\title{
HIPERPLASIA EPITELIAL FOCAL EN ESCOLARES SHUAR TAISHA- ECUADOR 2016
}

\section{FOCAL EPITHELIAL HYPERPLASIA IN SCHOOLS SHUAR TAISHA - ECUADOR 2016}

\author{
Corral-Peñafiel Davíd ${ }^{1 *}$, Villavicencio-Caparó Ebingen ${ }^{2}$, Paredes-Andrade Claudio ${ }^{3}$, Bravo-Calderón Diego ${ }^{4}$, \\ Cordero-Arias Juan ${ }^{5}$ \\ 1 Odontólogo Independiente egresado de la Universidad Católica de Cuenca. Ecuador \\ ${ }^{2}$ Dr. Mg. Esp. Catedrático de la Facultad de Odontología de la Universidad Católica de Cuenca. Ecuador \\ ${ }^{3}$ Odontólogo Independiente egresado de la Universidad Católica de Cuenca. Ecuador \\ ${ }^{4} \mathrm{PhD}$. MSc. Director de Investigación de odontologia de la Universidad de Cuenca. Ecuador \\ ${ }^{5}$ Estudiante la Universidad Católica de Cuenca. Ecuador \\ *bicho_corral16@hotmail.com
}

\begin{abstract}
Resumen
OBJETIVO: Revelar los posibles desencadenantes en la enfermedad de la Hiperplasia Epitelial Focal (HEF). MATERIALES Y MÉTODOS: El presente es un estudio longitudinal, observacional descriptivo, los datos se obtuvieron en la parroquia Taisha, en la comunidad San José en los meses de junio a septiembre del 2016. La población fue de 210 estudiantes. RESULTADOS: El estudio nos indicó que las condiciones de vida precoces presentan una mayor prevalencia con esta patología. CONCLUSIONES: Las condiciones en las que viven los estudiantes en su comunidad representa un posible desencadenante para que se prolifere la enfermedad.
\end{abstract}

Palabras clave: Hiperplasia Epitelial Focal, condiciones de vida, prevalencia.

\begin{abstract}
AIM The objective of the investigation was to Reveal possible triggers in Focal Epithelial Hyperplasia disease. MATERIALS AND METHODS: The present study was a longitudinal, observational, descriptive study; data were obtained from at Taisha parish, in the community of San José from June to September 2016. The population was 210 students. RESULTS: The study show that early life conditions have a higher prevalence with this pathology. CONCLUSIONS: The conditions in which students live in their community represents a possible trigger for the disease to proliferate.
\end{abstract}

Key words: Focal Epithelial Hyperplasia, living conditions, prevalence.

\section{INTRODUCCIÓN}

La Hiperplasia Epitelial Eocal (HEF), también conocida como enfermedad de Heck, es una enfermedad benigna rara, descrita por primera vez en la literatura inglesa por Archard et al (1965) ${ }^{1}$ como lesiones orales múltiples que afectaban a niños pertenecientes a las tribus navajo y amerindias. América Latina, Estrada $(1956)^{1}$ describió la presencia de verrugas intra orales y lesiones papilomatosas en los indios Caramantas en Colombia. ${ }^{1}$ Soneira y Fonseca $(1964)^{2}$ describieron la lesión con sus variaciones clínicas, desde una pápula hasta múltiples lesiones papilomatosas en la mucosa oral, labios, entre otras regiones de la mucosa oral. Todavía sugieren que la enfermedad tenía una etiología viral. ${ }^{2}$ Es relativamente frecuente en los indios de América
Central y América del Sur; hay una clara prevalencia en los esquimales y reporta una incidencia mundial de cuatro pacientes por 100000 habitantes. La concentración de casos HEF en algunas áreas geográficas con presencia familiar sugiere influencias ambientales y predisposición genética en su etiología. ${ }^{3,4}$ La hiperplasia epitelial focal es más común en las mujeres que en los hombres, la proporción encontrada es tan alta como 5: 1. La causa exacta de la mayor incidencia en las mujeres no se conoce. ${ }^{3,5,9}$ Algunos autores han propuesto que esto podría estar relacionado con las condiciones de vida asociadas con las mujeres en algunos grupos étnicos. ${ }^{6} \mathrm{Sin}$ embargo, la enfermedad de Heck se considera principalmente una enfermedad infecciosa causada por el virus del papiloma humano (VPH), especialmente 13 y 32 subtipos. ${ }^{1,2}$ Los fac- 
tores asociados descritos en la literatura incluyen la presencia de un gen recesivo, la inmunosupresión, el traumatismo, el tabaco de mascar, ${ }^{4,6}$ la deficiencia de vitamina $\mathrm{K},{ }^{4,6}$ la higiene oral deficiente y las condiciones sociales deplorables, ${ }^{4,6}$ pero no hay evidencia científica que afirme tales asociaciones. Por contacto sexual; ${ }^{4,6}$ En su lugar el contacto doméstico o el contacto con el ambiente pueden ser la principal fuente de infección. ${ }^{4,6}$ Clínicamente, la enfermedad se caracteriza por la presencia de múltiples pápulas y nódulos sésiles y suaves, de 1 a $10 \mathrm{~mm}$ de diámetro, que presentan un color similar a la mucosa circunyacente y por lo general afectan a los labios, la mucosa bucal y la lengua. ${ }^{2,4}$ Las lesiones pueden cambiar de tamaño durante meses a años. ${ }^{6}$ La hiperplasia epitelial focal se considera que tiene dos formas clínicas distintas: pápulo nodular y papilomatosa. La variante pápulo nodular es más común, las lesiones tienden a ser de color rosa y superficie lisa. Esta variante ocurre generalmente en la mucosa bucal y / o labial, y las comisuras de la boca. La variante papilomatosa de HEF es menos común y suele aparecer en la mucosa masticatoria como la lengua y la encía adherida. Las lesiones de este tipo son de color blanco y tienen una superficie rugosa áspera. ${ }^{6} \mathrm{El}$ diagnóstico se basa en criterios clínicos, junto con la ayuda de biopsias para identificar la enfermedad y diferenciarse de otras enfermedades bucales. El tratamiento es usualmente innecesario ya que la mayoría de las lesiones regresionan espontáneamente y no hay tendencia a la transformación maligna. Por lo tanto, el manejo de las lesiones de HEF sólo es necesario para fines estéticos o funcionales. ${ }^{1,7}$ Por lo tanto, si es necesario, debe ser extirpado quirúrgicamente y enviado a un laboratorio para diagnóstico definitivo. Teniendo en cuenta la baja frecuencia de la hiperplasia epitelial focal, y los informes sobre esta entidad en el Ecuador, se decidió llevar a cabo este estudio, con el objetivo de revelar los posibles desencadenantes de la enfermedad de HEF, lo que aumentará el conocimiento sobre esta enfermedad.

\section{MATERIALES Y MÉTODOS}

El presente es un estudio transversal, observacional descriptivo. Los datos se obtuvieron en la parroquia Taisha, en la comunidad San José en los meses de junio a septiembre del 2016. La población fue de 210 estudiantes comprendidos en las edades de 5 a 20 años de edad. Se solicitó los consentimientos informados y asentimientos informados de los estudiantes menores de edad para poder iniciar el presente estudio. Las condiciones para examinar fueron, por medio de un sillón portátil, el examinador detrás del paciente, los exámenes se hicieron en luz natural en la hora del recreo, el instrumental que se utilizó fue, un espejo bucal, guantes mascarilla y gorro, hubo un colaborador que anotaba los datos. La observación, se inició con la inspección desde los carillos y lengua, se diagnosticaba la presencia-ausencia de la hiperplasia epitelial focal o no, posteriormente de aquello se realizó la entrevista. Luego se utilizó el programa SPSS 20.0 para el análisis de los datos obtenidos.

\section{RESULTADOS}

Los resultados obtenidos en el estudio fueron:

La Tabla. 1 demuestra que existió un mayor predominio de Hiperplasia Epitelial de Focal en el Sexo Femenino que en el sexo masculino.

La Tabla. 2 indica que existió mayor cantidad de casos de la Hiperplasia Epitelial Focal en los estudiantes que consumen agua de tubería a comparación de los niños que toman agua de rio o agua de lluvia.

La Tabla. 3 revela que los estudiantes viven en casas construidas por concreto presentaron una mayor prevalencia de presentar la hiperplasia epitelial focal, seguido por los estudiantes que viven en casa de madera, siendo los último los que vivían en casas típicas.

La Tabla. 4 muestra que los niños con un mayor índice de desnutrición mostraron mayor prevalencia de tener la hiperplasia epitelial focal, en comparación con los niños que presentaban sobrepeso que no adquirieron la enfermedad.

La Tabla 5 nos indica que el mayor porcentaje de la enfermedad se desarrolló en la etapa de la niñez seguida de la infancia y se va disminuyendo desde la adolescencia a la adultez.

\section{DISCUSIÓN}

El presente estudio se realizó en la zona Amazónica del Ecuador la cual es conocida por el país como una zona de difícil acceso debido a que su única vía de ingreso es aérea. El estudio e realizó en la escuela "Los Ángeles" de la comunidad San José de la parroquia Taisha, los resultados se obtuvieron por medio del programa estadístico SPSS 22.0, en el cual se determinarón varios factores relacionados a la HEF y la caries.

De acuerdo a la figura 1 Determina que en el sexo femenino existe un mayor predominio de la HEF con un $11,70 \%$ corroborando lo encontrado por Harris en Colombia en el año 2016 donde indicó que el sexo femenino representaba el 63,64\% (8). Así como el estudio realizado por Pérez en México 2010, que esta patología es predominante en el género femenino. ${ }^{9}$

Con relación a la figura 2 y 3 manifestan que las condiciones vida que, las personas cercanas a la comunidad que viven en condiciones precarias tienen una menor prevalencia con respecto a los estudiantes que viven en condiciones más aceptables, éstos datos no concuerdan con lo encontrado en autores como Giovanni Corrado en el 2010, donde indica que a mayor condición precaria tiene un mayor riesgo de poseer la enfermedad. ${ }^{5}$

Respecto a la figura 4 habla sobre el factor nutricional que poseen los estudiantes, el estudio evidencio que a mayor 


\begin{tabular}{|c|c|c|c|c|c|c|}
\hline & & \multicolumn{5}{|c|}{ TIPO DE AGUA } \\
\hline & & & $\begin{array}{c}\text { AGUA DE } \\
\text { RÍOO }\end{array}$ & $\begin{array}{l}\text { AGUA DE } \\
\text { LLUV|A }\end{array}$ & $\begin{array}{l}\text { AGUA DE } \\
\text { TUBERIAA }\end{array}$ & TOTAL \\
\hline \multirow{4}{*}{ Prevalencia } & SANO & $\begin{array}{l}\text { Recuento } \\
\% \text { dentro de }\end{array}$ & 21 & 22 & 147 & 190 \\
\hline & & tipo de agua & $91,30 \%$ & $91,70 \%$ & $89,10 \%$ & $89,60 \%$ \\
\hline & CON HEF & Recuento & 2 & 2 & 18 & 22 \\
\hline & & $\begin{array}{l}\% \text { dentro de } \\
\text { tipo de agua }\end{array}$ & $8,70 \%$ & $8,30 \%$ & $10,90 \%$ & $10,40 \%$ \\
\hline \multirow[b]{2}{*}{ Total } & & Recuento & 23 & 24 & 165 & 212 \\
\hline & & $\begin{array}{l}\text { \% dentro de } \\
\text { tipo de agua }\end{array}$ & $100,00 \%$ & $100,00 \%$ & $100,00 \%$ & $100,00 \%$ \\
\hline
\end{tabular}

Tabla 1. Relación con el Agua

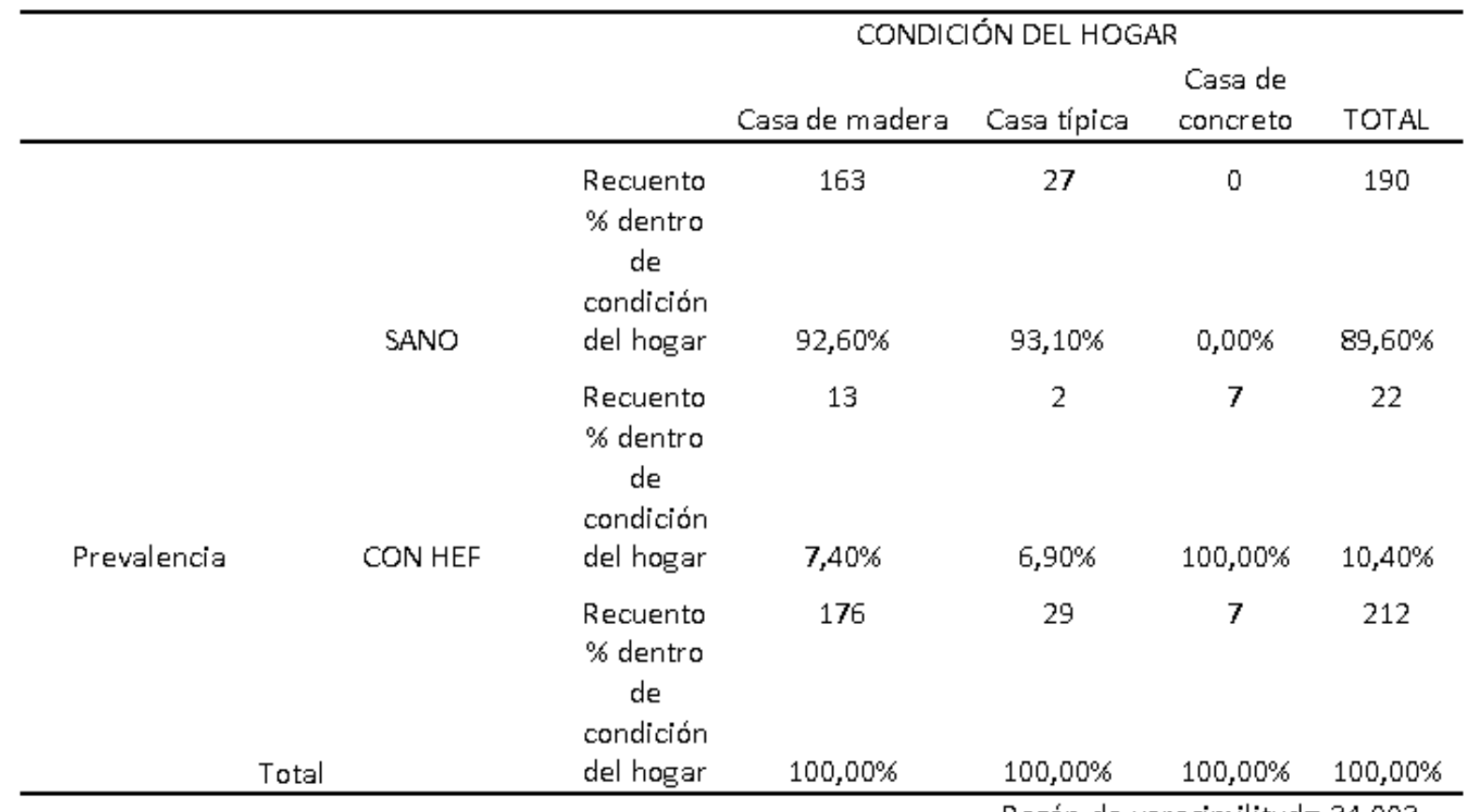

Tabla 2. Relación a la condición del hogar 


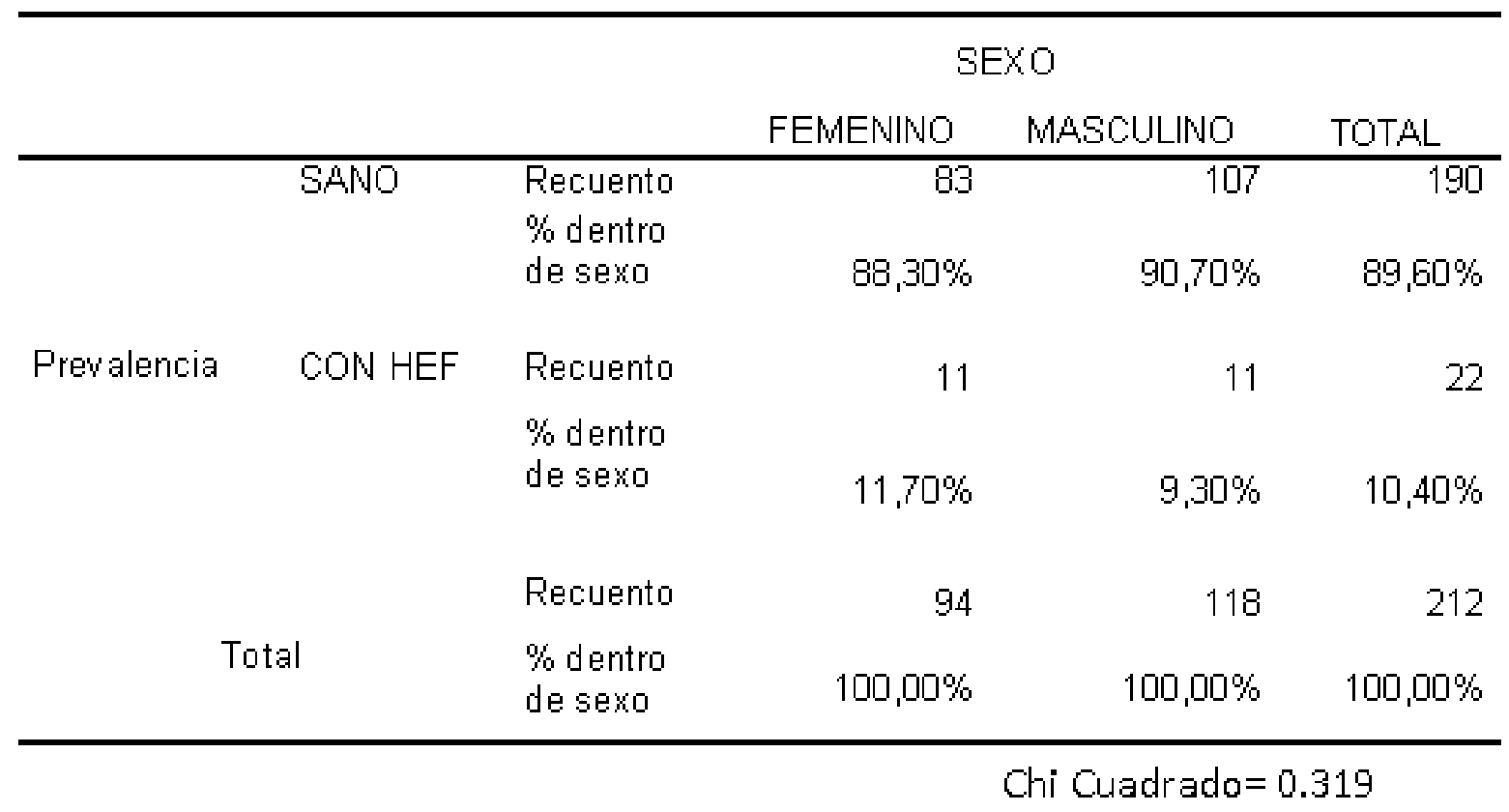

Tabla 3. Relación a la etapa de vida

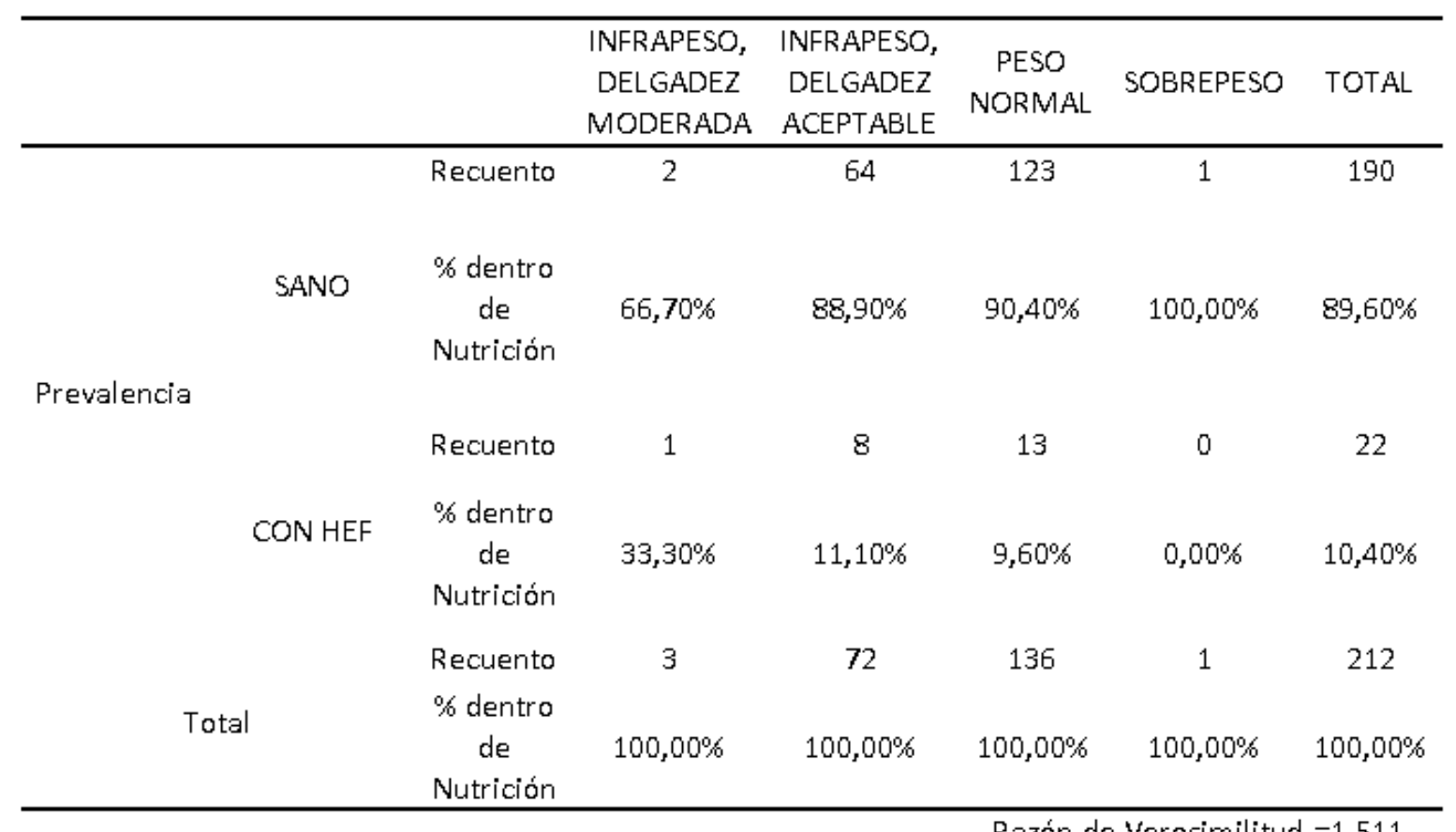

Tabla 4. Relación al estado nutricional 


\begin{tabular}{|c|c|c|c|c|c|c|c|}
\hline & & & \multicolumn{4}{|c|}{ ETAPA DE VIDA } & \multirow[b]{2}{*}{ TOTAL } \\
\hline & & & INFANTE & NINO & ADOLESCENTE & $\begin{array}{l}\text { ADULTO } \\
\text { JOVEN }\end{array}$ & \\
\hline \multirow{3}{*}{ Prevalencia } & SANO & $\begin{array}{l}\text { Recuento } \\
\% \text { dentro } \\
\text { de Etapa } \\
\text { devilda }\end{array}$ & $76,50 \%$ & $88,30 \%$ & $92,90 \%$ & $100,00 \%$ & $89,60 \%$ \\
\hline & \multirow[b]{2}{*}{$\begin{array}{l}\text { CON } \\
\text { HEF }\end{array}$} & Recuento & 4 & 12 & 6 & 0 & 22 \\
\hline & & $\begin{array}{l}\text { \% dentro } \\
\text { de Etapa } \\
\text { devida }\end{array}$ & $23,50 \%$ & $11,70 \%$ & $7,10 \%$ & $0,00 \%$ & $10,40 \%$ \\
\hline \multirow{2}{*}{\multicolumn{2}{|c|}{ Total }} & Recuento & 17 & 103 & 85 & 7 & 212 \\
\hline & & $\begin{array}{l}\text { \% dentro } \\
\text { de Etapa } \\
\text { devida }\end{array}$ & $100,00 \%$ & $100,00 \%$ & $100,00 \%$ & $100,00 \%$ & $100,00 \%$ \\
\hline
\end{tabular}

Tabla 5. Relación a las etapas de vida

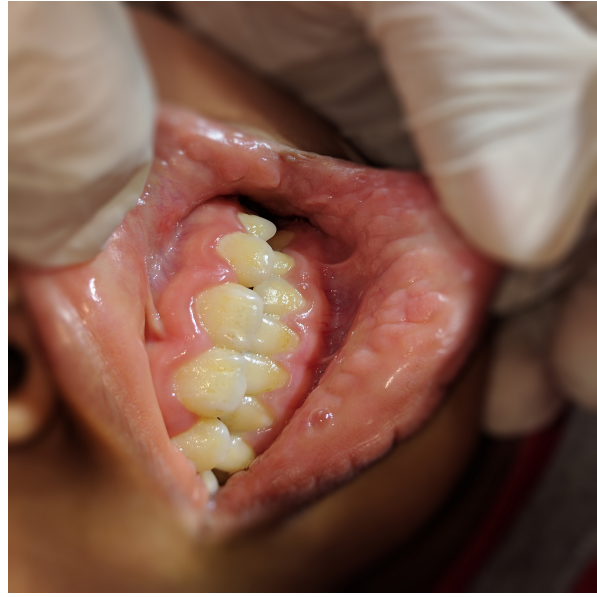

Fig. 1.

desnutrición (delgadez moderada) mayor probabilidad de presentar la HEF con un $33.3 \%$, éste resultado concuerda con lo expresado por Flores ${ }^{11}$ realizado en Venezuela donde indicó que el $25 \%$ de los casos presenta desnutrición. ${ }^{10}$

Respecto a la edad de aparición de la HEF, como se observa en la figura 5 donde la prevalencia en niños es de un $11.70 \%$, lo cual concuerda con el estudio realizado por Harris, ${ }^{8}$ al igual que Pérez ${ }^{10}$ que demostraron que el $90 \%$ de los casos analizados fueron niños. Otro estudio elaborado por Ahmed K en el 2013 demostró con una población mayor, que el $40 \%$ de los casos estudiados fueron niños. ${ }^{1}$

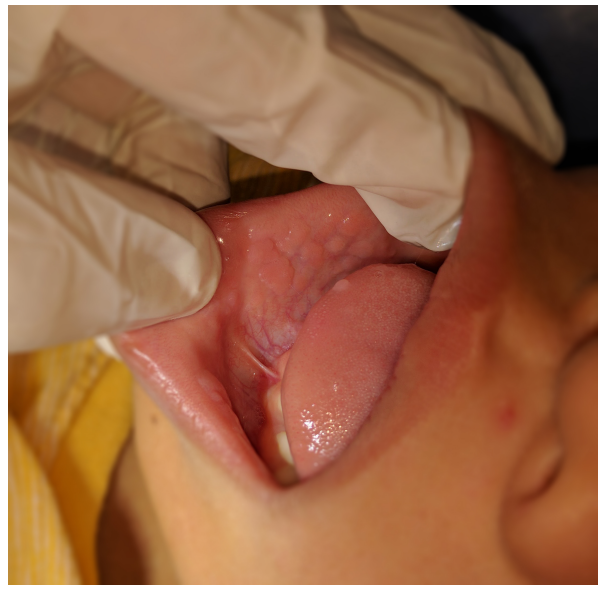

Fig. 2 .

\section{CONCLUSIONES}

Analizando todos los resultados del presente estudio se pudo observar que, el predominio de la enfermedad de HEF se establece con mayor prevalecía en el sexo femenino, durante la niñez y con un mayor grado de desnutrición (delgadez moderada).

Las condiciones en las que viven los estudiantes en su comunidad representan un posible desencadenante para que se prolifere la enfermedad. Así como el consumo del agua entubada donde indica que las condiciones sanitarias no son las indicadas en la comunidad. 


\section{AGRADECIMIENTO}

YUS IRMASTI TAISHA

DIOS TE BENDIGA TAISHA

Conflicto de intereses y financiamiento Los autores declaran no tener conflicto de intereses, haber cumplido con los requisitos de autoría y haber autofinanciado este artículo.

\section{Referencias}

1 Corrado G Haroyan E. Hiperplasia epitelial focal (Enfermedad de Heck): Búsqueda bibliográfica de los últimos 12 meses. Asignatura de Anatomía Patológica General y Bucal, URJC [Internet]. 2010. [Cited 18 jan 2017] 1-5pp .

2 Holgado F, Barbero M. Presentación del monográfico: validación de contenido desde metodologías cualitativas y cuantitativas. Acción Psicológica. 2014; 10 (2):1-2.Documento disponible

3 Pérez R. Pila Peláez M. Pila Peláez R. Focal Epithelial Hyperplasia. A Case Report. Medisur. 2014 [Cited 18 jan 2017] 100-105 pp. Documento disponible

4 Valdés González J. Solis Cartas U. Muñoz Balbín M. Epithelial Focal Hyperplasia or Heck Disease. Case presentation. Medisur. 2016 [Cited 18 jan 2017] 767-771 pp. $307-$ 15.Documento disponible

5 Castro L.A. Leite de Castro J.G. Lopez A.D. Focal Epithelial Hyperplasia (Heck's Disease) in a 57-Year-Old Brazilian Patient: A Case Report and Literature Review. J Clin Med Res. 2016 [Cited 18 jan 2017346-350pp. Documento disponible

6 Rosa LN. Gedoz L. Hildebrand LC. Hiperplasia Epitelial Focal: ¿por qué Enfermedad de Heck? [Internet] Avances en Estomatología. 2003. [Cited 18 jan 2017] 239-247pp. 110.Documento disponible

7 Said A.K. Leao J.C. Fedele S. Focal epithelial hyperplasia - an update. J Oral Pathol Med. 2013. [Cited 18 jan 2017] 435-442pp. Documento disponible

8 Alsadat Hashemipour M. Shoryabi A. Adhami S. Extensive Focal Epithelial Hyperplasia, Case Report. Iranian Medicine. 2010. [Cited 18 jan 2017] 48-52 ppDocumento disponible

9 Harris J. Carmona M. Diaz A Efectividad de la terapia con ácido tricloroacético en el tratamiento de lesiones en la hiperplasia epitelial focal. Medigraph. 2016 [Cited 18 jan 2017] 243-247 pp. Documento disponible

10 Perez A. Pino G.Lopez M. Hiperplasia epitelial focal: Actualidades y tratamiento. Revista Mexicana de Cirugía Bucal y Maxilofacial. 2010. [Cited 18 jan 2017] 111-115 pp. Documento disponible

11 Flores L. Silvio S. Concetta D. hiperplasia epitelial focal (Enfermedad de Heck): Presentación de un caso. Carabobo.2012 [Cited 18 jan 2017] 1-4 pp (1).
Recibido: 15 de Junio de 2016

Aceptado: 14 de Julio de 2016 